3 BÖHM, H., FREYER, J., CLAASSEN, M., HARTH, W., and BAUER, T: 'Second harmonic power generation from GaAs-IMPATT diodes at $210 \mathrm{GHz}$, Int. J. Infrared Millim. Waves, 1998, 19, (4), pp. $587-$ 593

4 EISELE, H.: 'Efficient second-harmonic power extraction from GaAs TUNNETT diodes above $200 \mathrm{GHz}$, Electron. Lett., 1998, 34, (13), pp. $1324-1326$

5 LIEBIG, D., and SCHÜNEMANN, K.: "Cellular automaton particle simulation and sensitivity analysis of GaAs-MITATT-diodes for operation at $200 \mathrm{GHz}$, Int. J. Electron. Commun. (AEÜ), 1998, 52, (5), pp. 329-334

6 GAUL, L., and CLAASSEN, M.: 'Pulsed high-power operation of $\mathrm{p}^{+} \mathrm{pnn}^{+}$-avalanche diodes near avalanche resonance for $\mathrm{mm}$-wave oscillators', IEEE Trans., 1994, ED-41, (8), pp. 1310-1318

7 FREYER, J., TSCHERNITZ, M., GROTHE, H., and HARTH, w.: 'GaAs transit time devices for frequencies above $140 \mathrm{GHz}$. Proc. 3rd Int Workshop on Terahertz Electronics, Zermatt, Switzerland, 1995, pp. $17-26$

8 BENZ, C., and FREYER, J.: 'Pulsed GaAs-IMPATT oscillator up to $200 \mathrm{GHz}$. Proc. 23rd Int. Conf. Infrared and Millimeter Waves, Colchester, UK, 1998, pp. 34-36

9 BAUER, T., and FREYER, J.: 'Steady-state simulation of mm-wave multiple device oscillators'. Proc. Int. Workshop on Millimeter Waves, Orvieto, Italy, 1996, pp. 145-147

\section{Extraction of noise parameters of transistor using a spectrum analyser and $50 \Omega$ noise figure measurements only}

\section{A. Lázaro and L. Pradell}

A method for measuring the four noise parameters of a transistor in the microwave range using a configuration based on a conventional spectrum analyser is presented. In contrast to previous methods, it requires wideband $50 \Omega$ noise-figure measurements only. The method features an accuracy similar to that of noise figure meters, at a much higher measurement speed and lower cost.

Introduction: A method for measuring the four transistor noise parameters (NPs), based on the determination of the intrinsic noise-matrix of the transistor from $50 \Omega$ noise-figure measurements only $\left(\mathrm{F}_{50}\right.$ technique), was recently proposed [1], demonstrating a relevant accuracy improvement compared to conventional tunerbased methods. In [1], the noise receiver was composed of a lownoise amplifier (LNA), a Yig filter, a down-converter (DC) with a local oscillator, and a noise-figure meter (NFM). The spectrum analyser (SA) is a lower cost alternative, since it integrates all the above functions (except for the LNA), and has a much higher measurement speed $[2,3]$. A problem when measuring noise with an SA is the poor linearity of its power-detector (including the log-amplifier) compared to that of an NFM [4]. Consequently, hot and cold noise power measurements $\left(P_{H O T}, P_{\text {COLD }}\right.$, respectively) with an SA are inaccurate (typically, noise-figure uncertainty is $\pm 0.4 \mathrm{~dB}[2])$, in particular whenever the $Y$-factor $\left(Y=P_{H O X} / P_{C O L D}\right)$ is large, i.e. the measured noise-figure is small. This situation is encountered when measuring NPs by using an SA in combination with a tuner, since some tuner states must fall near the optimum source reflection coefficient, corresponding to a minimum transistor noise-figure. The result is an inaccurate determination of the device NPs.

While the NP determination based on an SA is of interest due to its simplicity and reduced cost, improvements in accuracy are expected if the effects of poor linearity in the SA detector were minimised. The purpose of this Letter is to propose a new method for measuring the four NPs of a transistor, that combines a conventional $\mathrm{SA}$ with the $\mathrm{F}_{50}$ technique [1]. The method minimises the unwanted effects of detector linearity because:

(i) the measured $Y$-factor is smaller (higher noise-figure) than with tuner-based methods, since only $50 \Omega$ noise-figure measurements are made;

(ii) a new robust fitting algorithm is used to discard poor noisefigure measurements;

(iii) a large number of measurements are made to enhance frequency redundancy in the $\mathrm{F}_{50}$ technique.
The proposed method does not require any special, expensive instruments (DC, NFM, tuners), but only a conventional SA. Moreover, the receiver NPs are determined from $50 \Omega$ noise-figure measurements only, by making a reasonable assumption. The results demonstrate an accuracy comparable to that obtained by using $\mathrm{F}_{50}$ with a dedicated DC plus an NFM. Furthermore, they show an improved accuracy with respect to tuner-based methods. Experimental verification up to $26 \mathrm{GHz}$ is given.

Measurement method: The receiver is composed of the cascade connection of a commercial LNA and an SA (HP 70000). To calibrate the receiver noise, a matched noise source (HP 346-K01, with $\mathrm{ENR} \simeq 15 \mathrm{~dB}$ ) is connected to its input, hot and cold noise powers delivered to the receiver are measured over the whole range $(2-22 \mathrm{GHz})$, and the receiver noise-figure $\left(F_{R E C}\right)$ is computed at every frequency by using the expression

$$
F_{R E C}=\frac{\frac{P_{C O L D}}{\mu\left(\Gamma_{\left.S C, \Gamma_{R}\right)}\right.} \cdot\left(T_{H O T}-T_{C O L D}\right)}{\left(\frac{P_{H O T}}{\mu\left(\Gamma_{S H}, \Gamma_{R}\right)}-\frac{P_{C O L D}}{\mu\left(\Gamma_{S C,}, \Gamma_{R}\right)}\right) \cdot T_{0}}+1-\frac{T_{C O L D}}{T_{0}}
$$

where $T_{H O T}$ is the source hot temperature (known from the calibrated ENR data), $T_{C O L D}$ is the room temperature, $\Gamma_{S H}$ and $\Gamma_{S C}$ are hot and cold source reflection coefficients, respectively, $\Gamma_{R}$ is the receiver input reflection coefficient, $\mu\left(\Gamma_{S}, \Gamma_{R}\right)=\left(1-\left|\Gamma_{S}\right|^{2}\right) /\left|1-\Gamma_{S} \Gamma_{R}\right|^{2}$ is a mismatch factor, and $T_{0}=290 \mathrm{~K}$. Since the LNA in the receiver front-end is a well matched and basically unilateral device, it can be assumed that its equivalent noise-wave sources $b_{N 1}, b_{N 2}$ (in a scattering noise-matrix representation), are uncorrelated $\left(\overline{\left|b_{N 1} b_{N 2}^{*}\right|^{2}}=0\right.$ ) and $\overline{\left|b_{N 1}\right|^{2}} \simeq 0$ [5]. Under this hypothesis, the receiver NPs are functions of the normalised output noise power $\left(\overline{\left|b_{N 2}\right|^{2}} /\left|S_{21}\right|^{2}\right)$ only:

$$
\begin{gathered}
R_{n} \simeq \frac{Z_{0}}{4} \cdot\left(\overline{\left.\frac{\left|b_{N 2}\right|^{2}}{\left|S_{21}\right|^{2}}\right) \cdot\left|1+S_{11}\right|^{2}}\right. \\
F_{\text {min }} \simeq 1+\left(\overline{\frac{\left|b_{N 2}\right|^{2}}{\left|S_{21}\right|^{2}}}\right) \cdot\left(1-\left|S_{11}\right|^{2}\right) \\
\Gamma_{o p t} \simeq S_{11}^{*} \simeq \Gamma_{R}^{*}
\end{gathered}
$$

where $S_{11}, S_{21}$ are the receiver $S$-parameters. Since the LNA is basically unilateral and the SA is well matched, it is reasonable to assume that $S_{11} \simeq \Gamma_{R}$. The quantity $\left(\overline{\left|b_{N 2}\right|^{2}} /\left|S_{21}\right|^{2}\right.$ is computed using [6]

$$
F_{R E C}=1+\left[\left(1-\left|S_{11}\right|^{2}\right)+\frac{\left|\Gamma_{S C}-S_{11}^{*}\right|^{2}}{1-\left|\Gamma_{S C}\right|^{2}}\right] \cdot \frac{\overline{\left|b_{N 2}\right|^{2}}}{\left|S_{21}\right|^{2}}
$$

where $F_{R E C}$ is given by eqn. 1 . To complete the receiver calibration, the receiver NPs are computed by substituting $\overline{\left|b_{N 2}\right|^{2}} /\left|S_{21}\right|^{2}$ into eqns. 2 and 3 . To increase the measurement speed without degrading the measurement accuracy, small resolution and video bandwidths (RBW $=1 \mathrm{MHz}$, VBW $=3 \mathrm{KHz}$, respectively), as well as a high averaging (20) and short sweep time (1 s) are selected. The transistor is inserted, and its noise figure $\left(F_{T R T}\right)$ measured in the same way by using eqn. 1 , with $\Gamma_{I N}$ (transistor input reflection coefficient) instead of $\Gamma_{R}$, and the Friis' formula. The measured $F_{T R T}$ is used to estimate seven relevant polynomial coefficients that describe the transistor intrinsic noise matrix elements $C_{i j}^{I N T}\left(C_{i j}^{I N T}\right.$ $\left.=C_{i j}{ }^{0}+C_{i j}{ }^{1} f+\ldots\right)$ by applying the $\mathrm{F}_{50}$ technique [1]. In contrast to an NFM, the SA performs a large number $N$ of measurements (more than 100 in the frequency range), so the inherent frequencyredundancy of the $F_{50}$ technique is strongly enhanced. This feature, in combination with a low measured $Y$-factor, and the use of a robust error function (Lorentzian function $\Sigma_{i}\left(\log \left(1+0.5 \cdot \mid F_{\text {measured }}-\right.\right.$ $\left.\left.\left.F_{\text {compued }}^{i}\right|^{2}\right), i=1, \ldots, N\right)$ to discard possible outliers (bad noise-figure measurements) in the fitting algorithm, compensates for the undesired effects of a poor detector linearity. Moreover, the linear range of the measurement is optimised if the SA reference level (top of screen) is set $4-5 \mathrm{~dB}$ above the measured trace. The calculation of coefficients has two steps. First an overdetermined system (eqns. 5-10 in [1]) is solved for the most sensitive coefficients $\left(C_{22}{ }^{0}, C_{22}{ }^{1}, \operatorname{Re}\left(C_{12}{ }^{0}\right), \operatorname{Re}\left(C_{12}{ }^{1}\right)\right)$, neglecting the rest. Then $C_{11}{ }^{0}, C_{11}{ }^{1}$, $\operatorname{Im}\left(C_{12}{ }^{0}\right)$ are optimised using a modified simplex method Finally, $C_{22}{ }^{0}, C_{22}{ }^{1}, \operatorname{Re}\left(C_{12}{ }^{0}\right), \operatorname{Re}\left(C_{12}{ }^{1}\right)$ are optimised. 
Experimental results: Fig. 1 compares the NPs of the receiver $(\mathrm{LNA}+\mathrm{SA})$ up to $22 \mathrm{GHz}$ obtained with a tuner-based method [7] to those obtained with the new method proposed here. In general the results are in a good agreement, but the new method is more robust to measurement errors than the tuner-based method. This feature can be observed from 6 to $12 \mathrm{GHz}$. In this range, the tuner-based method fails because the SA is producing large measurement errors for some (mismatched) tuner states, due to a poor detector linearity.
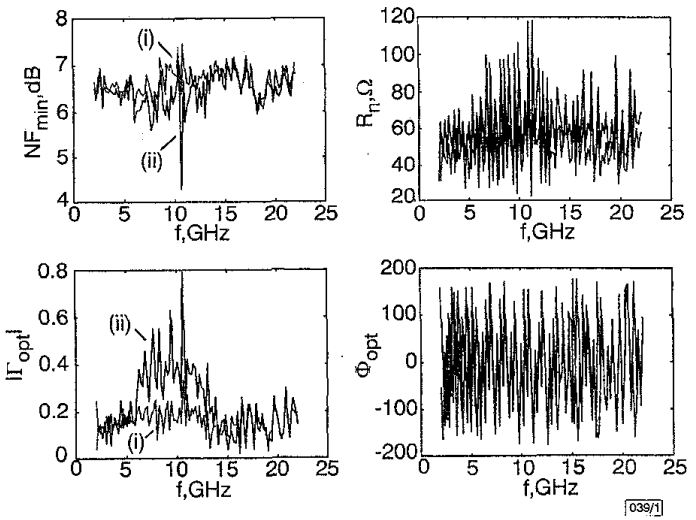

Fig. 1 Receiver noise parameters measured with spectrum analyser, using new method based on $F_{50}$ technique and tuner-based method proposed in [7]

(i) -...- new method based on $\mathrm{F}_{50}$ technique

(ii) tuner-based method in [7]
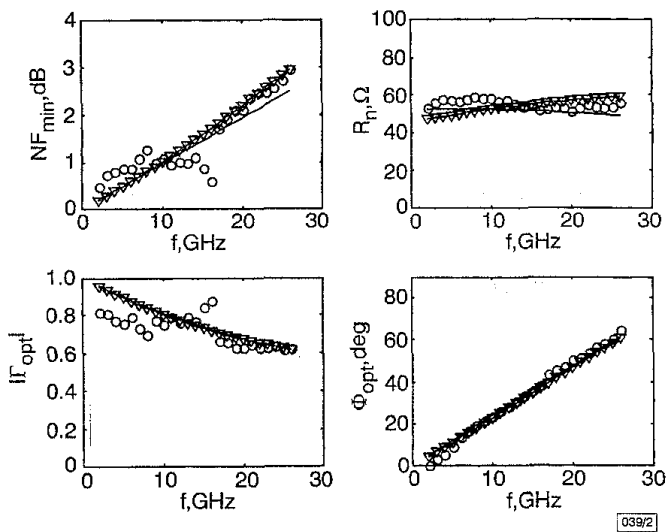

Fig. 2 HEMT noise parameters measured using new method based on $F_{50}$ technique and spectrum analyser, new method based on $F_{50}$ technique and noise figure meter, and tuner-based method proposed in [7]

$\nabla$ new method based on $F_{50}$ technique and spectrum analyser

0 tuner-based method [7]

Fig. 2 compares the NPS of an on-wafer HEMT measured with the method proposed here, using two different receivers: LNA+SA up to $22 \mathrm{GHz}$ and LNA+DC (HP 8971C) +NFM (HP 89708) up to $26 \mathrm{GHz}$, respectively. For the second configuration the NPs measured by using a tuner are also shown. To check the consistency of results for the LNA+SA configuration, the measured transistor intrinsic noise matrix is extrapolated from 22 to $26 \mathrm{GHz}$. The agreement between the three results is good at all frequencies except with the tuner-based method below $15 \mathrm{GHz}$ (corresponding $\left.\left|\Gamma_{o p t}\right|>0.7\right)$, for which $F_{\text {min }}$ and $\left|\Gamma_{\text {opt }}\right|$ are inaccurately measured. This result is in agreement with [7].

Conclusions: A fast and inexpensive method for the measurement of transistor NPs, based on a conventional spectrum analyser has been presented, and successfully applied to HEMTs. The effects of poor linearity in the SA detector are minimised because frequency redundancy is enhanced and only $50 \Omega$ noise figure measurements are required. Since noise powers are measured in a very short time ( 30 seconds per bias point), the calibration does not degrade with time, as with an NFM configuration (20min per bias point). Experimental results up to $26 \mathrm{GHz}$ are in very good agreement with those obtained with a noise figure meter. Moreover, they show an improved accuracy compared to tuner-based methods, in particular for critical measurement situation, a device $\left|\Gamma_{o p t}\right|$ close to 1 and very low measured noise figures.

Acknowledgment: This work has been supported by the research project TIC97-1129-C04-04 financed by the Spanish CICYT.

\section{(c) IEE 1998}

Electronics Letters Online No: 19981592

5 October 1998

A. Lázaro and L. Pradell (Polytechnic University of Catalunya (UPC), Dept. TSC, Campus Nord UPC, Modul D3, 08034 Barcelona, Spain)

\section{References}

1 LÁZARO, A., PRADELL, L., BELTRAån, A., and O'CALLAGHAN, J.M. 'Direct extraction of all four transistor noise parameters from $50 \Omega$ noise figure measurements', Electron. Lett., 1998, 32, (3), pp. 289 291

2 Hewlett-Packard Product Note 85719A-1: 'Maximising accuracy in noise figure measurements'. 1992

3 CROZAT, P., BOUTEX, C., CHAUbeT, M., DANELON, V., SYLVESTRE, A, and VERNET, A.: " $50 \Omega$ noise measurements with full receiver calibration without tuner', Electron. Lett., 1996, 32, (3), pp. 261262

4 Hewlett-Packard App. Note 57-1: 'Fundamentals of RF and microwave noise figure measurements'. Palo Alto, CA, July 1983

5 WERLING, T., BOURDEL, E., PASQUET, D., and BOUDIAF, A.: 'Determination of wave noise sources using spectral parametric modeling'. Proc. IEEE MTT-S Int. Microw. Symp. Dig., June 1997

6 DOBRowLSKI, J.A.: 'Introduction to computer methods for microwave circuit analysis and design' (Artech House, 1991)

7 LÁZARO, A., PRADELL, L., and O'CALLAGHAN, J.M.: 'Method for measuring noise parameters of microwave two-port', Electron. Lett., 1998, 34, (13), pp. 1332-1333

\section{High performance AIGaN/GaN HEMT with improved Ohmic contacts}

S.J. Cai, R. Li, Y.L. Chen, L. Wong, W.G. Wu, S.G. Thomas and K.L. Wang

Various metal films and rapid thermal annealing conditions were investigated to improve the Ohmic contact to AlGaN/GaN heterostuctures. Less than $1 \Omega \mathrm{mm}$ contact resistance has been obtained reproducibly. Our best contact resistivity reaches $0.039 \Omega \mathrm{mm}$, corresponding to a contact resistance of $5.38 \times 10^{-}$ ${ }^{8} \Omega \mathrm{cm}^{2}$. The fabricated high electron mobility transistors with a $0.25 \mathrm{um}$ length gate exhibit a cutoff frequency $f_{T}$ of $60 \mathrm{GHz}$ and an $f_{\max }$ of $100 \mathrm{GHz}$.

Significant progress has been made recently in both the high frequency and high power performances of GaN devices $[1-5]$, which mainly have come from improved material quality and advances in fabrication technology including the reduction of contact resistance [6-9]. For high power and low noise applications, the latter is of particular importance. Referring to Fig. 1, the saturation current density of $\mathrm{GaN}$ high electron mobility transistors (HEMTs) is plotted against conductivity ( $N_{s} \mu$ product). In addition to the material $N_{s} \mu$ product, the poor Ohmic contact is another main factor which limits the current density. For comparison, the data in Fig. 1 include those taken from HEMTs with high Ohmic contact resistivity (contact resistivity ranged from 2 to $12 \Omega$.mm as obtained from transmission line measurements). From this plot, it is reasonable to predict that nitride based HEMT's could achieve more than $2 \mathrm{~A} / \mathrm{mm}$ drain current with good Ohmic contact compared with the previously reported $1.71 \mathrm{~A} / \mathrm{mm}$, since the $N_{s} \mu$ product of $\mathrm{GaN}$ based heterojunction structures can reach $2.7 \times 10^{16} /$ V.s [3].

To improve the contact resistance, we have investigated several contact metal systems: Ti/Al, Ti/Al/Ni, Ti/Al/Pt, Ti/Al/Ni/Au and $\mathrm{Ti} / \mathrm{Al} / \mathrm{Pt} / \mathrm{Au}$, and different pre-cleaning methods before metal 